\title{
Bell diagonal states with maximal abelian symmetry
}

\author{
Dariusz Chruściński and Andrzej Kossakowski \\ Institute of Physics, Nicolaus Copernicus University \\ Grudziạdzka 5/7, 87-100 Toruń, Poland
}

\begin{abstract}
We provide a simple class of 2-qudit states for which one is able to formulate necessary and sufficient conditions for separability. As a byproduct we generalize well known construction provided by Horodecki et al. for $d=3$. It is hoped that these states with known separability/entanglement properties may be used to test various notions in entanglement theory.
\end{abstract}

PACS numbers: 03.67.Mn,03.65.Ud

\section{INTRODUCTION}

Physicists love solvable models, that is, models (or problems) for which one is able to answer all interesting questions. Clearly, solvable models are not generic. They are rather exceptional. However, they provide examples where one can study basic properties of generic models and to test various important notions. Usually, the 'solvability' or 'integrability' of the model is connected with the existence of the special symmetry. In many cases the presence of the symmetry enables one to simplify the analysis of the corresponding problems and very often it leads to much deeper understanding and the most elegant mathematical formulation of the corresponding physical theory.

In quantum information theory [1, 2] the idea of symmetry was first applied by Werner 3] to construct an important family of bipartite $\mathbb{C}^{d} \otimes \mathbb{C}^{d}$ quantum states which are invariant under the following local unitary operations: $\rho \rightarrow U \otimes U \rho(U \otimes U)^{\dagger}$, for any $U \in U(d)$ , where $U(d)$ denotes the group of unitary $d \times d$ matrices. Another family of symmetric states - so called isotropic states - 4 is governed by the following invariance rule $\rho=U \otimes \bar{U} \rho(U \otimes \bar{U})^{\dagger}$, where $\bar{U}$ is the complex conjugate of $U$ in some fixed basis in $\mathbb{C}^{d}$. Both families are 'solvable', that is, one can easily check wether a given $U \otimes U-$ or $U \otimes \bar{U}$-invariant state is separable or entangled.

In this paper we provide a class of states of a quantum composed system living in $\mathbb{C}^{d} \otimes \mathbb{C}^{d}$ for which one can easily check wether a given state is separable or entangled. It is well known that in general this problem is very hard [2, 5].

Our construction presented in the next section contains two ingredients:

1) we restrict to Bell diagonal states [6 9], and

2) we assume that $\rho$ is invariant under the action of the local group $U \otimes \bar{U}$, where $U$ belongs to the maximal commutative subgroup of $U(d)$ [10].

As a byproduct we provide a generalization of 'solvable' class of states constructed for $d=3$ by Horodecki et al. 11]. It is hoped that our 'solvable' family of states finds applications in Quantum Information Theory.

\section{DEFINITION OF THE 'SOLVABLE' CLASS}

Let $\{|0\rangle, \ldots,|d-1\rangle\}$ denotes an orthonormal basis in $\mathbb{C}^{d}$ and let $S: \mathbb{C}^{d} \rightarrow \mathbb{C}^{d}$ be a shift operator defined by

$$
S|k\rangle=|k+1\rangle, \quad(\bmod d)
$$

Consider now a simplex of Bell diagonal states [6-8] defined by (actually, one may provide more general definition, cf. [9])

$$
\rho=\sum_{m, n=0}^{d-1} p_{m n} P_{m n},
$$

where $p_{m n} \geq 0, \quad \sum_{m, n} p_{m n}=1$ and

$$
P_{m n}=\left(\mathbb{I} \otimes U_{m n}\right) P_{d}^{+}\left(\mathbb{I} \otimes U_{m n}^{\dagger}\right),
$$

with $U_{m n}$ being the collection of $d^{2}$ unitary matrices defined as follows

$$
U_{m n}|k\rangle=\lambda^{m k} S^{n}|k\rangle=\lambda^{m k}|k+n\rangle,
$$

where

$$
\lambda=e^{2 \pi i / d},
$$

and

$$
P_{00} \equiv P_{d}^{+}=\frac{1}{d} \sum_{i, j=0}^{d-1}|i i\rangle\langle j j|,
$$


denotes canonical maximally entangled state in $\mathbb{C}^{d} \otimes \mathbb{C}^{d}$. Actually, Bell diagonal states (2) belong to much more general class called circulant states [12] (see also [13]). Let us define

$$
\Pi_{0}=\frac{1}{d} \sum_{i=0}^{d-1}|i i\rangle\langle i i|,
$$

and

$$
\begin{aligned}
\Pi_{n} & =\left(\mathbb{I} \otimes S^{n}\right) \Pi_{0}\left(\mathbb{I} \otimes S^{n}\right)^{\dagger} \\
& =\frac{1}{d} \sum_{i=0}^{d-1}|i, i+n\rangle\langle i, i+n|,
\end{aligned}
$$

for $n=1, \ldots, d-1$. One has

$$
\Pi_{m} \Pi_{n}=\frac{1}{d} \delta_{m n} \Pi_{n},
$$

together with

$$
\Pi_{0}+\Pi_{1}+\ldots+\Pi_{d-1}=\frac{1}{d} \mathbb{I}_{d} \otimes \mathbb{I}_{d} .
$$

Proposition 1 ([10]) Any state $\rho$ defined by

$$
\rho=\sum_{m=0}^{d-1}\left[\mu_{m} \Pi_{m}+\nu_{m} P_{m 0}\right],
$$

satisfies

$$
U_{\mathbf{x}} \otimes \bar{U}_{\mathbf{x}} \rho=\rho U_{\mathbf{x}} \otimes \bar{U}_{\mathbf{x}}
$$

where

$$
U_{\mathbf{x}}=\exp \left(i \sum_{k=0}^{d-1} x_{k}|k\rangle\langle k|\right),
$$

and $\mathbf{x}=\left(x_{0}, \ldots, x_{d-1}\right) \in \mathbb{R}^{d}$.

Consider now the following family of states

$$
\rho=\sum_{i=1}^{d-1} \lambda_{i} \Pi_{i}+\lambda_{d} P_{d}^{+} .
$$

Note that (14) defines a subclass of (11). One has $\lambda_{1}, \ldots, \lambda_{d} \geq 0$, and $\lambda_{1}+\ldots+\lambda_{d}=1$. Clearly, the family of states (14) defines $(d-1)$-dimensional simplex with vertices $\Pi_{1}, \ldots, \Pi_{d-1}$ and $P_{d}^{+}$. One has the following

Note, that $\Pi_{1}, \ldots, \Pi_{d-1}$ define separable states (they are diagonal in the product basis $|i j\rangle=|i \otimes j\rangle)$. The family (14) can be fully characterized due to the following
Theorem $1 A$ state $\rho$ is PPT if and only if

$$
\lambda_{i} \lambda_{d-i} \geq \lambda_{d}^{2} .
$$

Moreover, a state $\rho$ is separable if and only if

$$
\lambda_{i} \geq \lambda_{d},
$$

for $i=1, \ldots, d-1$.

The proof of PPT condition (15) is easy and it is already contained in [10]. In the present paper we provide the proof of separability condition (16). Suppose that condition (16) is satisfied. Any state from the family (14) can be represented as the following convex combination

$$
\rho=d \lambda_{d} \widetilde{\rho}+\sum_{i=1}^{d-1}\left(\lambda_{i}-\lambda_{d}\right) \Pi_{i},
$$

where

$$
\widetilde{\rho}=\frac{1}{d}\left(\sum_{i=1}^{d-1} \Pi_{i}+P_{d}^{+}\right) .
$$

Now, it is well known that $\widetilde{\rho}$ is separable. Hence $\rho$ is separable being the convex combination of separable states $\widetilde{\rho}$ and $\Pi_{0}, \Pi_{1}, \ldots, \Pi_{d-1}$. To prove that separability implies (16) one needs to devise an appropriate entanglement witness.

\section{ENTANGLEMENT WITNESSES}

To define a border between separable and entangled states one needs an appropriate family of entanglement witnesses. Let us recall [14-16] (see laso [17-19]) that a Hermitian operator $W$ defined on a tensor product $\mathcal{H}=\mathcal{H}_{1} \otimes \mathcal{H}_{2}$ is called an EW iff 1$) \operatorname{Tr}\left(W \sigma_{\text {sep }}\right) \geq 0$ for all separable states $\sigma_{\mathrm{sep}}$, and 2) there exists an entangled state $\rho$ such that $\operatorname{Tr}(W \rho)<0$ (one says that $\rho$ is detected by $W$ ). It turns out that a state is entangled if and only if it is detected by some EW 14]. The simplest way to construct $\mathrm{EW}$ is to define $W=P+Q^{\Gamma}$, where $P$ and $Q$ are positive operators. It is easy to see that $\operatorname{Tr}\left(W \sigma_{\mathrm{sep}}\right) \geq 0$ for all separable states $\sigma_{\mathrm{sep}}$, and hence if $W$ is non-positive, then it is EW. Such EWs are said to be decomposable 17]. Note, however, that decomposable EW cannot detect PPT entangled state and, therefore, such EWs are useless in the search for bound entangled state. An EW which is not decomposable is called indecomposable (or non-decomposable). A PPT state $\rho$ is entangled iff there exists an indecomposable EW such that $\operatorname{Tr}(\rho W)<0$. 
Let us consider the following family of Hermitian operators

$$
W_{d, k}=(d-k) \Pi_{0}+\sum_{i=1}^{k} \Pi_{i}-P_{d}^{+},
$$

for $k=1,2, \ldots, d-1$. It is well known [20, 21] (see also [22, 23]) that for $k=1, \ldots, d-2, W_{d, k}$ defines an indecomposable EW, and $W_{d, d-1}$ is a decomposable EW. Actually, $W_{d, d-1}^{\Gamma} \geq 0$ and it corresponds to the reduction map, that is

$$
W_{d, d-1}=(1 \otimes R) P_{d}^{+},
$$

where $R(X)=\mathbb{I}_{d} \operatorname{Tr} X-X$. Now, it is evident from (9) and (10) that that the role of normalized projectors $\Pi_{1}, \ldots, \Pi_{d-1}$ is perfectly symmetric. Hence, for any permutation

$$
\pi:\{0,1, \ldots, d-1\} \longrightarrow\{\pi(1), \ldots, \pi(d-1)\}
$$

the new operator

$$
W_{d, k}^{\pi}=(d-k) \Pi_{0}+\sum_{i=1}^{k} \Pi_{\pi(i)}-P_{d}^{+},
$$

is again the legitimate EW. Note, however, that the property of (in)decomposability is not preserved for an arbitrary permutation, that is, $W_{d, k}^{\pi}$ might be decomposable/indecomposable even if $W_{d, k}$ is indecomposable/decomposable.

Now, if $\rho$ defined in (14) is separable then $\operatorname{Tr}\left(\rho W_{d, 1}^{\pi}\right) \geq 0$. One has

$$
\operatorname{Tr}\left(\rho W_{d, 1}\right)=\frac{1}{d}\left(\lambda_{1}-\lambda_{d}\right)
$$

and hence, separability of $\rho$ implies $\lambda_{1} \geq \lambda_{d}$. Taking an arbitrary permutation $\pi$ such that $\pi(1)=k$ one finds

$$
\operatorname{Tr}\left(\rho W_{d, 1}^{\pi}\right)=\frac{1}{d}\left(\lambda_{k}-\lambda_{d}\right)
$$

which finally proves (16).

Example 1 Consider a state $\rho$ defined in (14) with

$$
\lambda_{1}=\frac{\varepsilon}{N_{\varepsilon}}, \quad \lambda_{d-1}=\frac{1}{\varepsilon N_{\varepsilon}},
$$

and

$$
\lambda_{2}=\ldots=\lambda_{d-2}=\lambda_{d}=\frac{1}{N_{\varepsilon}}
$$

where $\varepsilon>0$, and the normalization factor

$$
N_{\varepsilon}=d\left(d-2+\varepsilon+\varepsilon^{-1}\right) .
$$

One has $\lambda_{1} \lambda_{d-1}=\lambda_{d}^{2}$ which shows that $\rho$ is PPT for all $\varepsilon>0$. However, the separability condition (16) is not satisfied unless $\varepsilon=1$. Hence, for $\varepsilon \neq 1$ a state (25) is PPT but entangled. Note that for $\varepsilon=1$ one has $\rho=\widetilde{\rho}$, where $\widetilde{\rho}$ is defined in (18).

Example 2 Consider now the special case of (14) defined by

$$
\lambda_{2}=\ldots=\lambda_{d-2}=\lambda_{d}
$$

where

$$
\begin{aligned}
\lambda_{1} & =\frac{\alpha}{N} \\
\lambda_{d-1} & =\frac{(d-1)^{2}+1-\alpha}{N}, \\
\lambda_{d} & =\frac{d-1}{N},
\end{aligned}
$$

with

$$
N=(d-1)(2 d-3)+1
$$

The parameter $\alpha \in\left[0,(d-1)^{2}+1\right]$. It is clear that for $d=3$ one recovers Horodecki construction [11]: $\Pi_{1}=$ $\sigma_{+}, \Pi_{2}=\sigma_{-}$, and

$$
\rho_{\alpha}=\frac{2}{7} P_{3}^{+}+\frac{\alpha}{7} \sigma_{+}+\frac{5-\alpha}{7} \sigma_{-},
$$

with $\alpha \in[0,5]$. It is well known that a state (30) is PPT for $\alpha \in[1,4]$. Moreover, it is separable for $\alpha \in$ $[2,3]$. Hence, for $\alpha \in[1,2) \cup(3,4]$ it is PPT entangled. Now, we perform a similar analysis for a generalized state. A state (14) defined by (28) is PPT if and only if $\lambda_{1} \lambda_{d-1} \geq \lambda_{d}^{2}$ which implies

$$
1 \leq \alpha \leq(d-1)^{2}
$$

Hence $\rho$ is separable if and only if $\lambda_{1}, \lambda_{d-1} \geq \lambda_{d}$ which is equivalent to

$$
d-1 \leq \alpha \leq(d-1)(d-2)+1 \text {. }
$$

Hence, for

$$
\alpha \in[1, d-1) \cup\left((d-1)(d-2)+1,(d-1)^{2}\right],
$$

a state is PPT but entangled. 


\section{GENERALIZED ISOTROPIC STATES} by

Consider now a simple generalization of (14) provided

$$
\rho=\sum_{i=0}^{d-1} \lambda_{i} \Pi_{i}+\lambda_{d} P_{d}^{+}
$$

that is, one adds an additional term ' $\lambda_{0} \Pi_{0}$ '. We stress that (34) still satisfies (12). However, in general Theorem 1 is no longer true. It is clear that PPT condition does not change: a state (34) is PPT iff the condition (15) is satisfied. Note that (16) implies separability. Indeed, one has

$$
\rho=d \lambda_{d} \widetilde{\rho}+\lambda_{0} \Pi_{0}+\sum_{i=1}^{d-1}\left(\lambda_{i}-\lambda_{d}\right) \Pi_{i},
$$

where $\widetilde{\rho}$ is defined in (18). Hence, it is a convex combination of separable states. Note however that now condition (16) is only sufficient but not necessary for separability of (34). The necessary conditions $\operatorname{Tr}\left(\rho W_{d, k}^{\pi}\right) \geq 0$ imply

$$
\frac{\lambda_{\pi(1)}+\ldots+\lambda_{\pi(k)}}{k} \geq \lambda_{d}-(d-k-1) \lambda_{0},
$$

for $k=1, \ldots, d-1$. In particular for $k=d-1$ one has

$$
\frac{\lambda_{1}+\ldots+\lambda_{d-1}}{d-1} \geq \lambda_{d}
$$

We stress that conditions (36) are necessary but not sufficient. For example if $d=3$ they give rise to

$$
\lambda_{1}, \lambda_{2} \geq \lambda_{3}-\lambda_{0}
$$

and

$$
\frac{\lambda_{1}+\lambda_{2}}{2} \geq \lambda_{3}
$$

In particular if $\lambda_{0} \geq \lambda_{3}$ then (38) is trivially satisfied and hence separability implies (39) which is much weaker than (16).

\section{Example 3 Taking}

$$
\lambda_{0}=\ldots=\lambda_{d-1}=\frac{1-\lambda_{d}}{d}
$$

one recovers well known isotropic state

$$
\rho=\frac{1-\lambda_{d}}{d^{2}} \mathbb{I}_{d} \otimes \mathbb{I}_{d}+\lambda_{d} P_{d}^{+} .
$$

Now, conditions (16) and (36) coincide and give rise to well known separability condition

$$
\lambda_{d} \leq \frac{1}{d+1}
$$

Hence, our generalized class (34) may be considered as a simple generalization of the isotropic state.

\section{CONLUSIONS}

We provide full characterization of the family of 2qudit states defined in (14). As a byproduct we introduce a 1-parameter class of states which generalizes Horodecki construction in $d=3$ [1]. It is shown that simple deformation of the original family provided by (34) is no longer 'solvable', i.e. we are not able to formulate complete list of necessary and sufficient conditions for separability. It is hoped that our class of states finds application in testing various notions in Quantum Information Theory.

\section{Acknowledgments}

This work was partially supported by the Polish Ministry of Science and Higher Education Grant No 3004/B/H03/2007/33.
[1] M. A. Nielsen and I. L. Chuang, Quantum computation and quantum information, Cambridge University Press, Cambridge, 2000.

[2] R. Horodecki, P. Horodecki, M. Horodecki and K.
Horodecki, Rev. Mod. Phys. 81, 865 (2009).

[3] R.F. Werner, Phys. Rev. A 40, 4277 (1989).

[4] M. Horodecki and P. Horodecki, Phys. Rev. A 59, 4206 (1999). 
[5] O. Gühne and G. Tóth, Phys. Rep. 474, 1 (2009).

[6] B. Baumgartner, B. Hiesmayr, and H. Narnhofer, Phys. Rev. A 74, 032327 (2006).

[7] B. Baumgartner, B. Hiesmayr, and H. Narnhofer, J. Phys. A: Math. Theor. 40, 7919 (2007).

[8] B. Baumgartner, B. Hiesmayr, and H. Narnhofer, Phys. Lett. A 372, 2190 (2008).

[9] R.F. Werner, J. Phys. A: Math. Gen. 34, 7081 (2001).

[10] D. Chruściński and A Kossakowski, Phys. Rev. A 74, 022308 (2006)

[11] P. Horodecki, M. Horodecki, and R. Horodecki, Phys. Rev. Lett. 82, 1056 (1999).

[12] D. Chruściński and A. Kossakowski, Phys. Rev. A 76, 032308 (2007).

[13] D. Chruściński and A. Pittenger, J. Phys. A: Math. Theor. 41, 385301 (2008).

[14] M. Horodecki, P. Horodecki, and R. Horodecki, Phys. Lett. A 223, 1 (1996).
[15] B. Terhal, Phys. Lett. A 271, 319 (2000); Linear Algebr. Appl. 323, 61 (2000).

[16] B. M. Terhal, Theor. Comput. Sci. 287, 313 (2002).

[17] M. Lewenstein, B. Kraus, J. I. Cirac, and P. Horodecki, Phys. Rev. A 62, 052310 (2000).

[18] M. Lewenstein, B. Kraus, P. Horodecki, and J. I. Cirac, Phys. Rev. A 63, 044304 (2001).

[19] B. Kraus, M. Lewenstein, and J. I. Cirac, Phys. Rev. A 65, 042327 (2002).

[20] K. Tanahashi and J. Tomiyama, Can. Math. Bull. 31, 308 (1988).

[21] H. Osaka, Linear Algebra Appl. 153, 73 (1991); 186 , 45 (1993).

[22] D. Chruściński, A. Kossakowski, Open Sys. Information Dyn. 14, 275 (2007).

[23] D. Chruściński and A. Kossakowski, J. Phys. A: Math. Theor. 41, 145301 (2008). 